\title{
Kulikovskiy-Sveshnikova-Beghin model of powder snow avalanches: Development and application
}

\author{
B. Turnbull, ${ }^{1,2}$ J. N. McElwaine, ${ }^{3}$ and C. Ancey ${ }^{4}$ \\ Received 20 February 2006; revised 13 June 2006; accepted 12 September 2006; published 8 February 2007.
}

[1] A simple theoretical model, the Kulikovskiy-Sveshnikova-Beghin (KSB) model, is outlined, describing the motion of a particle cloud moving down an incline. This model includes both the entrainment of surrounding ambient fluid and the entrainment of particles from the slope and is equally valid for Boussinesq and non-Boussinesq flows. However, this model can predict physically impossible densities when there is significant particle entrainment. Modifications to the model are proposed which eliminate this problem by including the entrained snow volume. With the modified model, physically realistic mean densities are predicted which have a significant impact on the Richardson number-dependent ambient entrainment. The improvements are illustrated by comparing analytical solutions to the original and the modified KSB equations for the case of a particle cloud traveling on a slope of constant angle, with constant ambient fluid and particle entrainment. Solving the modified model numerically, predictions are compared with data from several large powder snow avalanches at the Swiss Vallée de la Sionne avalanche test site. The modified KSB model appears to capture the dynamics of the avalanche front well; however, problems remain with relating the theoretical geometry to a real avalanche geometry. The success of this model in capturing the front dynamics shows that with careful assumptions that reflect the physics, it is possible to describe aspects of complex flows such as powder snow avalanches with simple models.

Citation: Turnbull, B., J. N. McElwaine, and C. Ancey (2007), Kulikovskiy-Sveshnikova-Beghin model of powder snow avalanches: Development and application, J. Geophys. Res., 112, F01004, doi:10.1029/2006JF000489.

\section{Introduction}

[2] Powder snow avalanches are a dramatic, naturally occurring example of a flow driven by the density difference between a particle suspension and the surrounding fluid. There are many further examples of particle driven gravity currents not only in geophysics, for example pyroclastic ash flows from volcanoes and turbidity currents of silt on the ocean floor, but also in industry. The presence of particles, kept in suspension by turbulence in the interstitial fluid, increases the mean density of the suspension compared with the surrounding ambient fluid, providing a driving force. In a powder snow avalanche, this driving density difference is maintained by the entrainment of particles from the snow cover which counteracts dilution of the suspension through air entrainment.

[3] There are several aspects of powder snow avalanches which require special consideration. In particular, the high density difference between snow particles and the surround-

\footnotetext{
${ }^{1}$ Swiss Federal Institute of Snow and Avalanche Research, WSL, Davos Dorf, Switzerland.

${ }^{2}$ Now at Department of Applied Mathematics and Theoretical Physics, University of Cambridge, Cambridge, UK.

${ }^{3}$ Department of Applied Mathematics and Theoretical Physics, University of Cambridge, Cambridge, UK.

${ }^{4}$ Ecole Polytechnique Fédérale de Lausanne, Lausanne, Switzerland.

Copyright 2007 by the American Geophysical Union. 0148-0227/07/2006JF000489\$09.00
}

ing air means that, even for snow particle clouds with solid concentrations of only a few percent by volume, the Boussinesq approximation [Boussinesq, 1903] is not valid and the cloud is in a non-Boussinesq regime. That is, the inertia due to the density differences cannot be neglected since the snow particles carry a significant proportion of the suspension's momentum (for $1 \%$ concentration by volume, the particles carry $90 \%$ of the momentum).

[4] The Kulikovskiy-Sveshnikova-Beghin (KSB) model is a simple theoretical model for the motion of a particle cloud on an incline, incorporating entrainment of both ambient fluid and particles. The Boussinesq approximation is not made in the model's derivation, making it applicable to nonBoussinesq clouds such as powder snow avalanches. First introduced in this form by Ancey [2004], the KSB model originates from the work of Kulikovskiy and Sveshnikova [1977] who obtained equations of mass, momentum, volume and Lagrangian kinetic energy balances. Beghin [1979] developed his work, neglecting energy considerations, by introducing a slope angle dependence to supplement the density ratio dependence of ambient entrainment assumed by Kulikovskiy and Sveshnikova [1977].

[5] Ancey [2004] developed these theories further by comparing Beghin's slope-angle-dependent ambient entrainment assumption to a growth rate governed by overall Richardson number [Turner, 1973], consistent with the entrainment assumption proposed for inclined plumes by Ellison and Turner [1959]. The Richardson number is 
the ratio of the potential energy to the kinetic energy of a parcel of fluid. At large Richardson numbers the restoring effect of gravity across an interface dominates the inertial effects and the interface is stable. Entrainment at the interface increases with decreasing stability of the interface and so we expect the entrainment rate to increase with decreasing Richardson numbers. Both slope-angle-dependent and Richardson number-dependent entrainment functions were tested by Ancey [2004] with data from finite volume laboratory releases on an incline with particle entrainment (unpublished data obtained by Beghin, reproduced by Ancey, Revol and Clément) and data from the 25th February 1999 avalanche at the Vallée de la Sionne avalanche test site [Dufour et al., 2000]. For both cases (except for high concentration laboratory releases), the Richardson numberdependent entrainment function could reproduce the velocities and volumes well compared with the slope-angle-dependent entrainment function [Ancey, 2004]. A detailed overview of the literature, discussing a range of modeling approaches, is given by Ancey [2004].

[6] In some respects the simplicity of the KSB model might be considered a step back from the more sophisticated powder snow avalanche models that are currently being developed [Sampl, 1993; Scheiwiller et al., 1987; Naaim and Gurer, 1998]. However, even fully three-dimensional models must make many assumptions and choices for turbulence closures and mass, volume and momentum exchanges within and between layers of the flow. These submodels are mostly not well verified in the parameter ranges appropriate for powder snow avalanches. The result is that many additional parameters must be chosen. However, several of these models have been calibrated and successfully applied to particular avalanche tracks.

[7] There are other, simpler models which vary subtly from the KSB formulation. For example Beghin and Olagne [1991] use thermal theory to find mass and momentum equations for a two- or three-dimensional buoyant cloud. This provides a similar framework to the KSB formulation, though importantly Beghin and Olagne [1991] make the assumption of no snow entrainment but include basal friction. This restricts the models applicability to the latter stages of the avalanche and contrasts with the KSB formulation where the inertia of entrained snow provides a retarding force much greater than the basal friction. Also closely related to the KSB model is the Fukushima and Parker [1990] formulation, which itself has been developed further by Gauer [1995]. These models include the original four equations, including energy considerations which increase their practical use. In the present work we are interested in a formulation where any assumptions can be straightforwardly tested and can be applied to both laboratory and field data. In this way we can directly understand the underlying physics.

[8] In section 5 of this paper, the KSB equations are derived from two-phase continuum theory. With careful assumptions, the KSB theory used throughout the paper is provided, which requires no additional closure assumptions. This derivation links the current work to future formulations of the KSB model. The objectives of this paper are to show what the KSB model can describe and predict; to develop the model, removing some deficiencies of earlier formulations; and to apply the model to further field data. To achieve these objectives, general analytical solutions to the KSB equations are found and evaluated for the case of a particle cloud flowing down an incline of constant slope angle, with constant particle and ambient fluid entrainment. The equations are solved numerically for a real avalanche track with varying slope angle, varying particle entrainment and with ambient entrainment a function of the overall Richardson number. In this way the field case presented by Ancey [2004] is reproduced and attention is drawn to some of the model's deficiencies; in particular, the unphysically large predicted densities. It is shown that by including the volume of entrained snow, physically possible densities are predicted, significantly affecting the Richardson number-dependent ambient entrainment. Analytical solutions to the modified equations are found and contrasted with the original analytical predictions.

[9] Predictions of the modified model are compared with data from the Vallée de la Sionne avalanche test site, operated by the Swiss Federal Institute of Snow and Avalanche Research (SLF). This is a field site where large powder snow avalanches can be artificially triggered with explosives to flow past sensors mounted on a mast [Dufour et al., 2000]. Video recordings of the avalanches, taken from two or three different locations, have been analyzed allowing the digital reconstruction of the avalanche surface at chosen time frames [Gruber, 2004; Vallet et al., 2004; Turnbull, 2006]. From these measurements, front velocity, average flow height, and avalanche volume data at each time frame can be found. One significant problem is that powder snow avalanches are very sensitive to the amount of entrained snow cover; a problem which is reflected in all models, however complex. Without high-quality data on entrained or entrainable snow cover the model cannot predict how far a powder cloud can travel or typical flow velocities.

\section{KSB Equations}

[10] The KSB model is an integral model for powder snow avalanches that has conservation equations for volume, mass and momentum. Here, the geometry of the model is introduced and the equations are derived from the arguments given by Ancey [2004]. The equations are more rigorously derived from the underpinning continuum theory in section 5 .

[11] The powder cloud is modeled as a half ellipse in longitudinal cross section with unit lateral width, i.e., the model is two-dimensional (see Figure 1). The axes of the ellipse are aligned with the slope, which is assumed to be locally flat over scales the size of the avalanche. The aspect ratio $k=h / l$, where $h$ is the height and $l$ the length, is assumed to be a function of the slope angle $\theta$ only. For a semiellipse, the volume per unit width is $V=\frac{\pi}{4} h l$. The cloud of mean density $\rho$ flows into ambient fluid (which is air in the case of a powder snow avalanche) of density $\rho_{a}$ and entrains a snow layer of density $\rho_{s}$ and depth $h_{n}$. A curvilinear coordinate system is used where $s$ is the arc length, that is the distance of the center of mass down the slope. The arc length $s$ is a function of the horizontal and vertical coordinates $x$ and $y$ and increases down the slope. Front velocity $u_{f}$ is related to the center-of-mass velocity $u$ by $u_{f}=u+\frac{1}{2} \frac{\mathrm{d} l}{\mathrm{~d} t}$. 


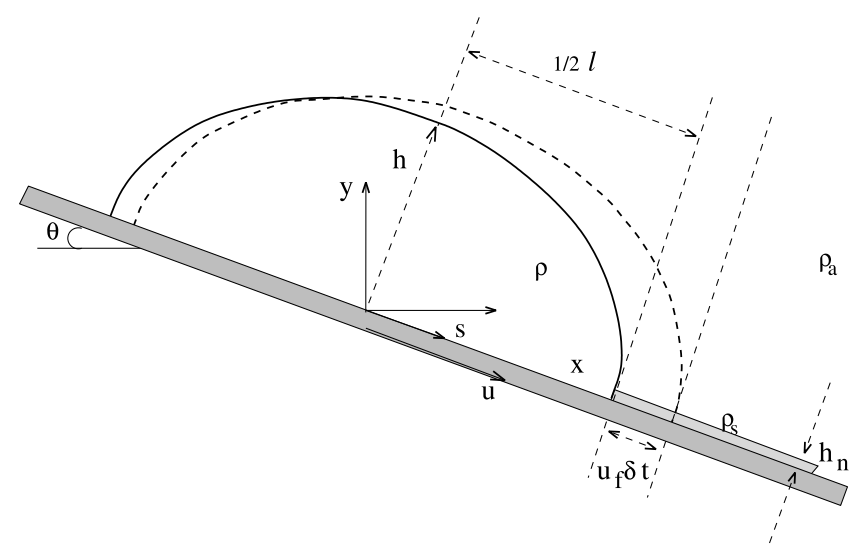

Figure 1. Schematic of the KSB model. The semiellipses represent the powder cloud at a time $t$ (solid outline) and at a time $t+\delta t$ (dashed outline).

[12] A volume equation is derived by assuming that the volume of entrained snow mass is small compared with the volume of entrained ambient air. From Turner [1973], the height of an inclined plume varies with time

$$
\frac{\mathrm{d} h}{\mathrm{~d} t}=u f(\mathrm{Ri}),
$$

where $f(\mathrm{Ri})$ determines the air entrainment. For the geometry in Figure 1 the height, $h$, is proportional to the square root of the volume, $\sqrt{V}$. Hence the volume growth is determined by an air entrainment coefficient $\alpha_{v}$ and the center-of-mass velocity $u$

$$
\frac{\mathrm{d} V}{\mathrm{~d} t}=\alpha_{v} u \sqrt{V},
$$

where $\alpha_{v}=f$ (Ri) $\sqrt{\pi / k}$ for the given geometry. Since $V$ has units of surface, $\alpha_{v}$ is dimensionless.

[13] A simplified mass or buoyancy equation is found by assuming that the settling velocity of the snow particles is very much smaller than mean downslope velocity of the cloud front. This is the case when the ratio of the terminal velocity of the particles to the flow velocity $v_{t} / u$ is small. This means that the model is not appropriate as the avalanche decelerates and deposition becomes important. To extend the model to this region, a turbulent kinetic energy equation modeling the effects of turbulence on particle sedimentation would be necessary, increasing the complexity. If the mass of air in the cloud is $m_{a}$ and the mass of snow in the cloud is $m_{s}$, the total cloud mass, $\rho V$, is the sum of the two, $\rho V=m_{a}+m_{s}$. The buoyancy of the cloud is defined as

$$
B=\left(\rho-\rho_{a}\right) V,
$$

which can be written in terms of the component masses of snow and air

$$
B=m_{a}+m_{s}-\rho_{a} V .
$$

The total volume flux is the sum of the volume fluxes of air into the cloud at the top surface, $q_{a}$, and snow into the cloud at the bottom surface, $q_{s}$, which gives

$$
\frac{\mathrm{d} V}{\mathrm{~d} t}=q_{s}+q_{a} .
$$

The mass fluxes of air and snow into the cloud are

$$
\frac{\mathrm{d} m_{a}}{\mathrm{~d} t}=\rho_{a} q_{a} \quad \text { and } \quad \frac{\mathrm{d} m_{s}}{\mathrm{~d} t}=\rho_{s} q_{s}
$$

respectively, where $\rho_{a}$ and $\rho_{s}$ are the corresponding densities of air and entrained snow. Differentiating equation (3) and substituting from the above definitions we have

$$
\frac{\mathrm{d} B}{\mathrm{~d} t}=\left(\rho_{s}-\rho_{a}\right) q_{s}
$$

Snow entrainment into the powder cloud is assumed to be characterized by the density $\rho_{s}$ and a depth $h_{n}$ which depends on the cloud's position on the slope (see Figure 1). This assumption has been observed to be reasonable over most of the track [Bozhinskiy and Losev, 1998] where the avalanche usually slides on an interface between layers in the snow pack. This erosion depth, $h_{n}$, can be measured from photogrammetry for avalanches or could be estimated from the snow stratigraphy. For a cloud with front velocity $u_{f}$, the volume flux of snow entrained into the cloud is $q_{s}=u_{f} h_{n}$. The buoyancy equation follows from equation (4) to give

$$
\frac{\mathrm{d} B}{\mathrm{~d} t}=\left(\rho_{s}-\rho_{a}\right) u_{f} h_{n}
$$

[14] The front velocity is a function of the center-of-mass velocity, which can be found from the geometry shown in Figure 1

$$
u_{f}=u\left(1+\frac{\alpha_{v}}{2 \sqrt{\pi k}}\right)
$$

[15] A momentum equation is derived assuming that the basal friction is small, which will be true at high Reynolds numbers, but not in the decelerating, deposition phase of the avalanche [Hogg and Woods, 2001]. (A typical Reynolds number for a powder snow avalanche in the transition zone with a height $\approx 20 \mathrm{~m}$, front velocity $\approx 50 \mathrm{~m} \mathrm{~s}^{-1}$, and density $\approx 10 \mathrm{~kg} \mathrm{~m}^{-3}$ has an order of magnitude $10^{8}$, and the basal friction can certainly be considered small). In real snow avalanches, it has often been observed that beneath the powder snow suspension there is a denser, fluidized layer of snow which has nonnegligible basal drag [Issler, 1998]. The KSB equations do not model the internal dynamics and can either model just the suspension part of the avalanche, or alternatively the dense fluidized layer can be considered part of the powder cloud. If the latter is the case, then the dense layer effectively introduces a phase lag in snow entrainment, since the snow is first entrained into the dense layer and later entrained from the dense layer into the powder cloud. 
[16] In addition, the direct pressure drag is assumed to be small compared to the force necessary to accelerate the entrained ambient air. The downslope component of gravitational force is $B g \sin \theta$. It is assumed that the effect of accelerating the ambient air close to the cloud can be included by the added mass coefficient $\chi$ [Batchelor, 1967] such that the effective inertial mass of the avalanche is

$$
M=B+(1+\chi) V \rho_{a}
$$

The added mass coefficient for an ellipse is derived in Appendix A, where we show that $\chi=k$. Although the added mass for a powder snow avalanche is small, it is important for the laboratory experiments used to calibrate the entrainment coefficients [Ancey, 2004] where the ambient fluid is water and not air. The momentum equation follows

$$
\frac{\mathrm{d}}{\mathrm{d} t}\left\{\left[B+(1+\chi) V \rho_{a}\right] u\right\}=B g \sin \theta .
$$

\subsection{Analytical Solutions}

[17] Analytical solutions are given in the appendix of Ancey [2004] in a different form. They are derived here to provide a comparison with the solutions presented in section 3.

[18] For the solution of equations (1), (5), and (8) each is written in a nondimensional form by posing

$$
\left(\tilde{s}, \tilde{\rho}_{a}, \tilde{u}\right)=\left(\frac{s}{L}, \frac{\rho_{a}}{\rho_{s}}, \frac{u}{\sqrt{L g}}\right),
$$

where $L$ is an arbitrary length scale, $\rho_{s}$ is the snow cover density and $g$ is the acceleration due to gravity. Changing variables from $t$ to $s, \frac{\mathrm{d}}{\mathrm{d} t}=\tilde{u} \frac{\mathrm{d}}{\mathrm{d} \tilde{s}}$, the equations can be written in terms of their spatial derivative. The nondimensional volume equation is therefore

$$
\frac{\mathrm{d} \tilde{V}}{\mathrm{~d} \tilde{s}}=\alpha_{v} \sqrt{\tilde{V}}
$$

which can be written

$$
2 \frac{\mathrm{d}}{\mathrm{d} \tilde{s}} \sqrt{\tilde{V}}=\alpha_{v}
$$

Under the condition that the entrainment parameter $\alpha_{v}$ is a function of slope arc coordinate, $\tilde{s}$, only, equation (10) can be integrated directly with a virtual origin $\tilde{s}_{0 V}$ which satisfies the initial condition, $\tilde{V}\left(\tilde{s}_{0 V}\right)=0$ giving

$$
\tilde{V}=\frac{1}{4}\left[\int_{\tilde{s}_{0 V}}^{\tilde{s}} \alpha_{v}\left(\tilde{s}^{\prime}\right) \mathrm{d} \tilde{s}^{\prime}\right]^{2} .
$$

[19] The buoyancy equation (5) is similarly nondimensionalized with respect to the length and density scales in equation (9). With the variable $\eta$ defined such that

$$
\eta=\tilde{h}_{n}\left(1-\tilde{\rho}_{a}\right)\left(1+\frac{\alpha_{v}}{2 \sqrt{\pi k}}\right),
$$

the buoyancy equation becomes

$$
\frac{\mathrm{d} \tilde{B}}{\mathrm{~d} \tilde{s}}=\eta
$$

This is integrated assuming the effective entrained snow $\eta$ is a function of slope arc coordinate, $\tilde{s}$, only, to give

$$
\tilde{B}=\int_{\tilde{s} 0 B}^{\tilde{s}} \eta\left(\tilde{s}^{\prime}\right) \mathrm{d} \tilde{s}^{\prime} .
$$

Here, the virtual buoyancy origin $\tilde{s}_{0 B}$ satisfies the condition $\tilde{B}\left(\tilde{s}_{0 B}\right)=0$.

[20] Including the added mass of ambient air accelerated around the avalanche, the nondimensional effective inertial mass of the avalanche is

$$
\tilde{M}=\tilde{B}+\beta \tilde{V},
$$

where $\beta=(1+\chi) \tilde{\rho}_{a}$. Usually the momentum equation can be converted to an energy equation and integrated. Given the nondimensional kinetic energy $\tilde{E}=\frac{1}{2} \tilde{M} \tilde{u}^{2}$, its derivative can be written

$$
\frac{\mathrm{d} \tilde{E}}{\mathrm{~d} \tilde{s}}=\tilde{B} \sin \theta-\frac{1}{2} \tilde{u}^{2} \frac{\mathrm{d} \tilde{M}}{\mathrm{~d} \tilde{s}}
$$

The first term on the right hand side is the driving gravitational force which is counteracted by a term dependent on the change in effective inertial mass. This energy equation shows that as the particle cloud entrains mass along its path, energy is transferred to this additional mass, accelerating it but retarding the cloud. Energy is also dissipated at a rate $\frac{1}{2} \tilde{u}^{2} \frac{\mathrm{d} M}{\mathrm{~d} \tilde{s}}$, since no basal or aerodynamic drag is included in the model and energy lost in mixing the entrained matter is the only dissipation mechanism.

[21] Equation (8) can be more simply integrated if, rather than converting to an energy equation, the momentum equation (8) is multiplied by $\tilde{M}$. Nondimensionalizing and writing in terms of slope arc coordinate, $\tilde{s}$, gives

$$
\frac{1}{2} \frac{\mathrm{d}}{\mathrm{d} \tilde{s}}(\tilde{M} \tilde{u})^{2}=\tilde{M} \tilde{B} \sin \theta,
$$

where $\tilde{M} \tilde{u}$ is the cloud momentum. Thus

$$
\tilde{u}=\frac{1}{\tilde{M}} \sqrt{2 \int_{\tilde{s}_{0 u}}^{\tilde{s}} \tilde{M}\left(\tilde{s}^{\prime}\right) \tilde{B}\left(\tilde{s}^{\prime}\right) \sin \left[\theta\left(\tilde{s}^{\prime}\right)\right] \mathrm{d} \tilde{s}^{\prime}},
$$

with the virtual origin $\tilde{s}_{0 u}$ chosen such that $\tilde{u}\left(\tilde{s}_{0 u}\right)=0$. Note that the above analytical solutions for volume (equation (11)), buoyancy (equation (14)) and velocity (equation (17)) are general; particle, ambient entrainment and the slope angle can all be functions of slope arc coordinate $\tilde{s}$. Analytical solutions will be more complicated to find if the particle and ambient entrainment, $\eta$ and $\alpha_{v}$, are functions of the dynamic variables.

[22] Cloud volume, density and velocity can be found explicitly from the above solutions with the assumption of 


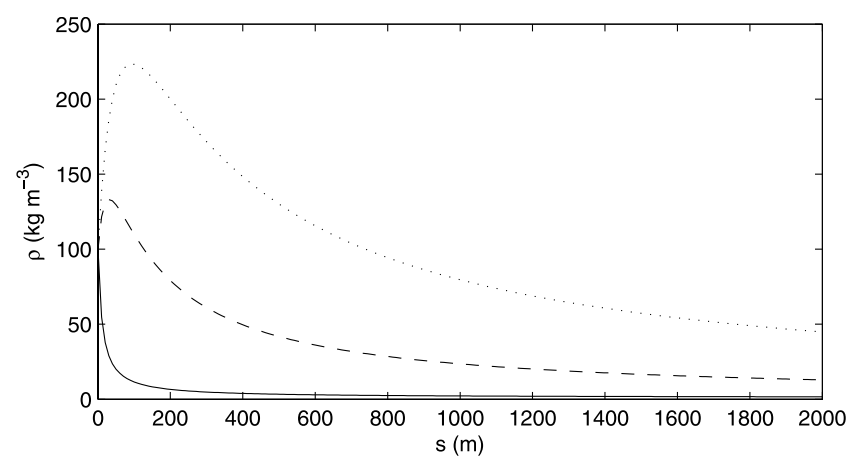

Figure 2. Variation of powder cloud mean density, $\rho$, with slope arc position, $s$, for three different volumetric growth rates: solid line, $\alpha_{v}=0.5$; dashed line, $\alpha_{v}=0.1$; dotted line, $\alpha_{v}=0.05$.

constant slope angle $\theta$, and constant particle and ambient entrainment, $\eta$ and $\alpha_{v}$ respectively. The volume solution is simply integrated to give

$$
\tilde{V}=\left[\frac{\alpha_{v}\left(\tilde{s}-\tilde{s}_{0 V}\right)}{2}\right]^{2} .
$$

Using this volume solution and given buoyancy and density are related by $\tilde{B}=\left(\tilde{\rho}-\tilde{\rho}_{a}\right) \tilde{V}$, the powder cloud density follows from equations (11) and (14)

$$
\tilde{\rho}=\tilde{\rho}_{a}+\frac{4 \eta\left(\tilde{s}-\tilde{s}_{0 B}\right)}{\alpha_{v}^{2}\left(\tilde{s}-\tilde{s}_{0 V}\right)^{2}} .
$$

[23] This density solution, equation (19), is shown in Figure 2 for three different volumetric growth rates, $\alpha_{v}=$ $0.05,0.1$, and 0.5 . The solutions in Figure 2 use initial and ambient conditions appropriate for powder snow avalanches. The dimensional virtual origins, $s_{0 V}$ and $s_{0 B}$, and also the dimensional snow entrainment $\eta$, follow directly from their definitions, with choices of initial volume and initial density and entrained snow depth, $h_{n}$. Here we have chosen initial values; $V_{0}=10 \mathrm{~m}^{2}, \rho_{0}=100 \mathrm{~kg} \mathrm{~m}^{-3}, h_{n}=$ $0.4 \mathrm{~m}, \rho_{s}=150 \mathrm{~kg} \mathrm{~m}^{-3}$, and $k=0.4$, which are the correct magnitude for a powder snow avalanche. The resulting dimensional $s_{0 V}, s_{O B}$, and $\eta$ have been evaluated for each $\alpha_{v}$ in Table 1 .

[24] In the cases shown in Figure 2, the densities become unphysically large, especially after short distances where the powder cloud is small. For example, the maximum mean density predicted is over $200 \mathrm{~kg} \mathrm{~m}^{-3}$, which is larger than the density of the entrained snow cover.

Table 1. Virtual Origins $s_{0 V}, s_{0 B}$, and the Snow Entrainment $\eta$ (Equation (12)) for Three Values of Air Entrainment Coefficient $\alpha_{v}=0.05,0.1,0.5^{\mathrm{a}}$

\begin{tabular}{lrrr}
\hline & \multicolumn{3}{c}{$\alpha_{v}$} \\
\cline { 2 - 4 } & 0.05 & 0.1 & 0.5 \\
\hline$\eta, \mathrm{kg} \mathrm{m}^{-2}$ & 60.8 & 62.1 & 72.7 \\
$s_{0 V}, \mathrm{~m}$ & -126 & -63.2 & -12.6 \\
$s_{0 B}, \mathrm{~m}$ & -0.162 & -0.159 & -0.135 \\
\hline
\end{tabular}

${ }^{\mathrm{a}}$ The initial conditions are $V_{0}=10 \mathrm{~m}^{2}, \rho_{0}=100 \mathrm{~kg} \mathrm{~m}^{-3}, h_{n}=0.4 \mathrm{~m}$, $\rho_{s}=150 \mathrm{~kg} \mathrm{~m}^{-3}$, and $k=0.4$; values are representative of powder snow avalanches.
[25] Substituting into equation (17) for the volume and buoyancy, equations (11) and (14), respectively, gives the powder cloud velocity as a function of slope arc position, $\tilde{s}$, only. With the assumptions of constant slope angle, $\theta$ and entrainment parameters $\eta$ and $\alpha_{v}$, the general solution is cumbersome. There is an arbitrary choice in the origin of $\tilde{s}_{0 u}$, and the solution is somewhat simpler if $\tilde{s}_{0 u}$ is taken to be 0 . The nondimensional cloud velocity is then

$$
\tilde{u}=\frac{\sqrt{\eta \tilde{s} f(\tilde{s}) \sin \theta}}{\tilde{M}(\tilde{s})}
$$

where the function $f(\tilde{s})$ is

$$
\begin{aligned}
f(\tilde{s})= & \frac{1}{8} \alpha_{v}^{2} \beta\left[\left(\tilde{s}-\tilde{s}_{0 V}\right)^{3}+\left(\frac{1}{3} \tilde{s}^{2}-\tilde{s} \tilde{s}_{0 V}+\tilde{s}_{0 V}^{2}\right)\left(\tilde{s}_{0 V}-4 \tilde{s}_{0 B}\right)\right] \\
& +2 \eta\left(\frac{1}{3} \tilde{s}^{2}-\tilde{s}_{0 B}+\tilde{s}_{0 B}^{2}\right)
\end{aligned}
$$

[26] Since $\beta=(1+\chi) \tilde{\rho}_{a}$ and $\tilde{\rho}_{a}$ is the ambient density scaled with the snow density, $\beta=(1+\chi) \rho_{a} / \rho_{s}$. In the limit where the snow cover is very much denser than the ambient air, the ratio $\rho_{a} / \rho_{s}$ is small and so $\beta$ is also small. In this high-density case, letting $\beta \rightarrow 0$, a relatively simple form of the cloud velocity, $\tilde{u}_{d}$, can be found

$$
\tilde{u}_{d}=\frac{\sqrt{6 \tilde{s}\left(\tilde{s}^{2}-3 \tilde{s} \tilde{s}_{0 B}+3 \tilde{s}_{0 B}^{2}\right) \sin \theta}}{3\left(\tilde{s}-\tilde{s}_{0 B}\right)} .
$$
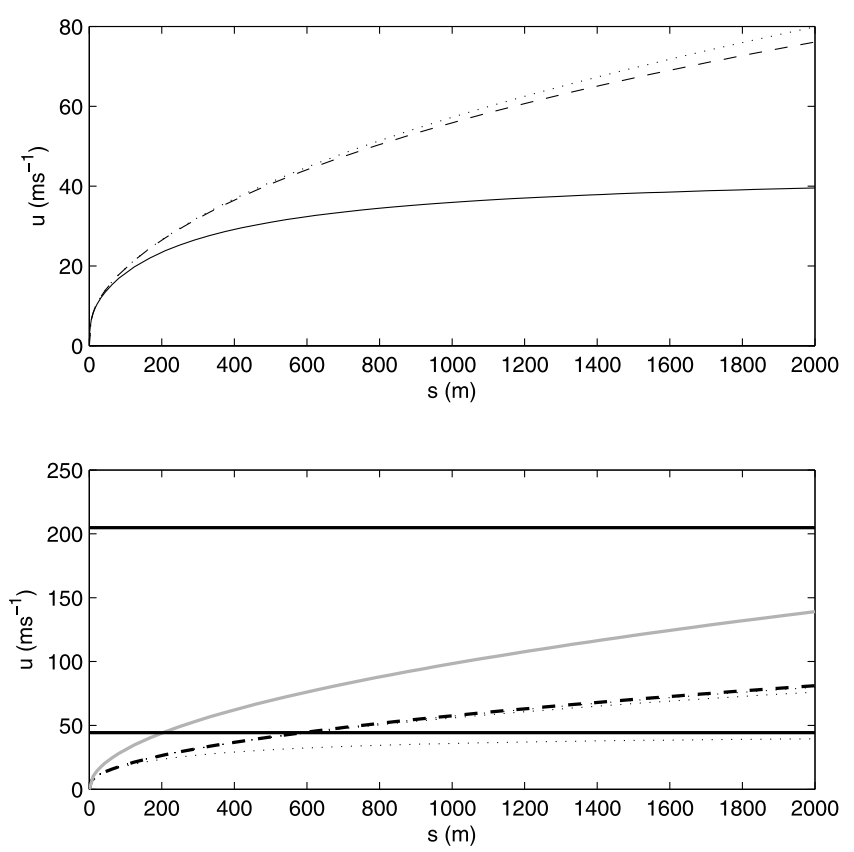

Figure 3. (top) Powder cloud velocities, $u$, as a function of slope arc position, $s$, on a flat slope for constant entrainment parameters given in Tables 1 and 2 (equation (20)): solid line, $\alpha_{v}=0.5$; dashed line, $\alpha_{v}=0.1$; dotted line $\alpha_{v}=0.05$. (bottom) Dotted lines, solution as above; dashed line, highdensity solution (equation (21)); the solid gray line, the small $s$ approximation (equation (22)); solid black lines, asymptotic solution as $s \rightarrow \infty$ for $\alpha_{v}=0.1, u \infty=205 \mathrm{~m} \mathrm{~s}^{-1}$ and for $\alpha_{v}=0.5, u \infty=44 \mathrm{~m} \mathrm{~s}^{-1}$ (equation (24)). 


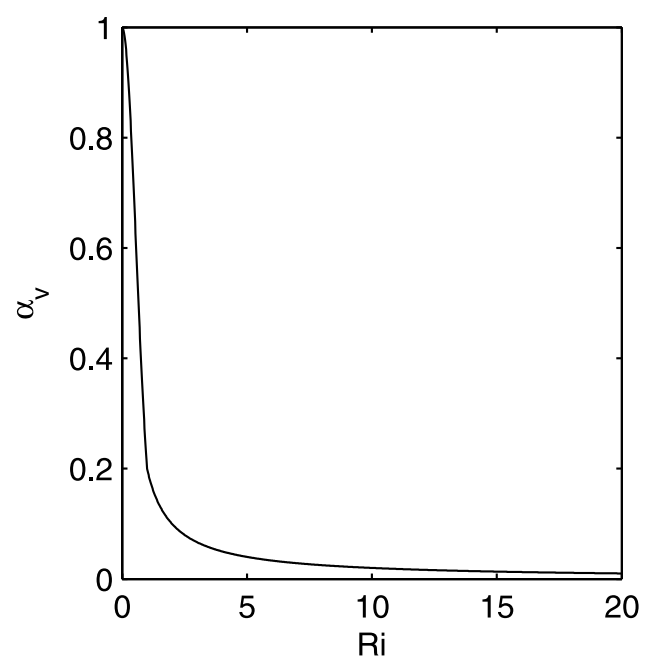

Figure 4. Entrainment parameter $\alpha_{v}$ as a function of Richardson number, Ri (equation (27)).

This solution is representative of a dense avalanche where the dominating process is particle entrainment. In this regime, drag due to the acceleration of entrained matter is much larger than basal friction. The general and highdensity solutions (equations (20) and (21), respectively) are plotted in Figures 3 and 4 for various values of volumetric growth rates, $\alpha_{v}=0.05,0.1$, and 0.5 . As for the cloud densities in Figure 2, the initial and ambient conditions are appropriate for powder snow avalanches, listed in Table 2 giving the virtual origins shown in Table 1 . In addition, for the semiellipse geometry shown in Figure 1, it can be shown that the added mass coefficient is equal to the ellipse's aspect ratio, $\chi=k$ (see Appendix A). Note that in Figure 3 there is a good coincidence of this high-density assumption (dashed line) with the dotted line showing the full solution with low air entrainment, $\alpha_{v}=0.05$. For the early part of the avalanche in particular, where the density, and thus Richardson number, is high, $\alpha_{v}=0.05$ is a typical value.

[27] All of the curves show the powder cloud accelerating sharply initially. This corresponds to the approximate solution for small values of $\tilde{s}$, which from the expansion of equation (20) is

$$
\tilde{u}_{s}=2 \sqrt{\frac{2 \eta \tilde{s}_{0 B} \sin \theta}{4 \eta \tilde{s}_{0 B}-\beta \alpha_{v}^{2} \tilde{s}_{0 V}^{2}}}+O\left(\tilde{s}^{3 / 2}\right) .
$$

This is perhaps more clearly written in terms of the initial density $\tilde{\rho}_{0}=\tilde{\rho}(0)$ when equation (22) can be rearranged to give

$$
\tilde{u}_{S}=\sqrt{\frac{2 \tilde{s} \sin \theta\left(1-\frac{\tilde{\rho}_{a}}{\tilde{\rho}_{0}}\right)}{\left(1+\chi \chi_{\tilde{\rho}_{0}}\right)}} .
$$

If the initial density $\rho_{0}$ is very low, $\rho_{0} \rightarrow \rho_{a}$ and $\tilde{\rho}_{0}-\tilde{\rho}_{a}$ approaches 0 . In this case the velocity $\tilde{u}_{s} \rightarrow 0$ since there is no driving density difference. Conversely, if $\rho_{0} \gg \rho_{a}$, then the velocity $\tilde{u}$ approaches a limiting value of $\sqrt{2 \tilde{s} \sin \theta}$.

[28] Over large distances the curve flattens and the cloud approaches a steady velocity, to leading order, which can be found from equation (20)

$$
\tilde{u}_{\infty} \rightarrow \sqrt{\frac{2 \eta \sin \theta}{\beta \alpha_{v}^{2}}}+O\left(\frac{1}{\tilde{s}}\right), \quad \text { as } \quad \tilde{s} \rightarrow \infty .
$$

This steady asymptotic velocity is independent of the initial conditions and only varies with ambient and particle entrainment and the slope angle.

\subsection{Numerical Results}

[29] The volume, mass and momentum KSB equations ((1), (5), and (8)) can be solved numerically for a real avalanche track given a track profile of $x$ and $y$, horizontal and vertical, coordinates along the axis of the avalanche (Figure 1). Initial conditions of the powder cloud volume, density and velocity, and the height and density of the entrained snow cover must be given.

[30] The equations were numerically solved in MATLAB using the ode45, Runge-Kutta [Riley et al., 1997] solver. There are several key differences between this approach and the approach of Ancey [2004], for example in smoothing the track profile points to provide the slope angle, $\theta$, as a continuous function of track position, $s$. In the work by Ancey [2004] the track profile points were interpolated and fitted with Legendre Polynomials where here, smoothing splines were fitted to the interpolated points, giving $x(s)$ and $y(s)$. A further difference is that here a simpler formulation for the entrained snow depth has been used. This was a piecewise linear function, which had little effect on results in comparison with smoother functions. In order to ensure

\begin{tabular}{|c|c|c|c|c|c|}
\hline & \multicolumn{5}{|c|}{ Avalanche } \\
\hline & Analytical & 200 & 509 & 628 & 726 \\
\hline Date & - & 25 Feb 1999 & 7 Feb 2002 & 19 Jan 2004 & 17 Feb 2005 \\
\hline Erosion depth $h_{n}, \mathrm{~m}$ & 0.4 & 1.0 & 0.1 & 0.1 & $0.1^{\mathrm{b}}$ \\
\hline Snow cover density $\rho_{s}, \mathrm{~kg} \mathrm{~m}^{-3}$ & 150 & 200 & 195 & 200 & $200^{\mathrm{b}}$ \\
\hline Slope angle $\theta$ & $30^{\circ}$ & - & - & - & - \\
\hline Added mass coefficient $\chi$ & 0.4 & - & - & - & - \\
\hline Shape factor $k$ & 0.4 & - & - & - & - \\
\hline Air density $\rho_{a}, \mathrm{~kg} \mathrm{~m}^{-3}$ & 1.04 & 1.04 & 1.04 & 1.04 & 1.04 \\
\hline Gravity $g, \mathrm{~m} \mathrm{~s}^{-2}$ & 9.81 & 9.81 & 9.81 & 9.81 & 9.81 \\
\hline Initial volume, $V_{0}, \mathrm{~m}^{2}$ & 10 & 100 & 100 & 100 & 100 \\
\hline Initial density $\rho_{0} \mathrm{~kg} \mathrm{~m}^{-3}$ & 100 & 100 & 100 & 100 & 100 \\
\hline Initial velocity $u_{0}, \mathrm{~m} \mathrm{~s}^{-1}$ & 0 & 1 & 1 & 1 & 1 \\
\hline
\end{tabular}
errors in calculating the slope angle do not accumulate,

Table 2. KSB Initial and Ambient Conditions ${ }^{\mathrm{a}}$

${ }^{a}$ Erosion depths are given to the nearest $0.05 \mathrm{~m}$. The air density is calculated from the 1976 standard atmosphere for an altitude of $2000 \mathrm{~m}$ asl at a sea level temperature of $3^{\circ} \mathrm{C}$, which gives a $2000 \mathrm{~m}$ asl temperature of $-10^{\circ} \mathrm{C}$.

${ }^{\mathrm{b}}$ Estimated values. 

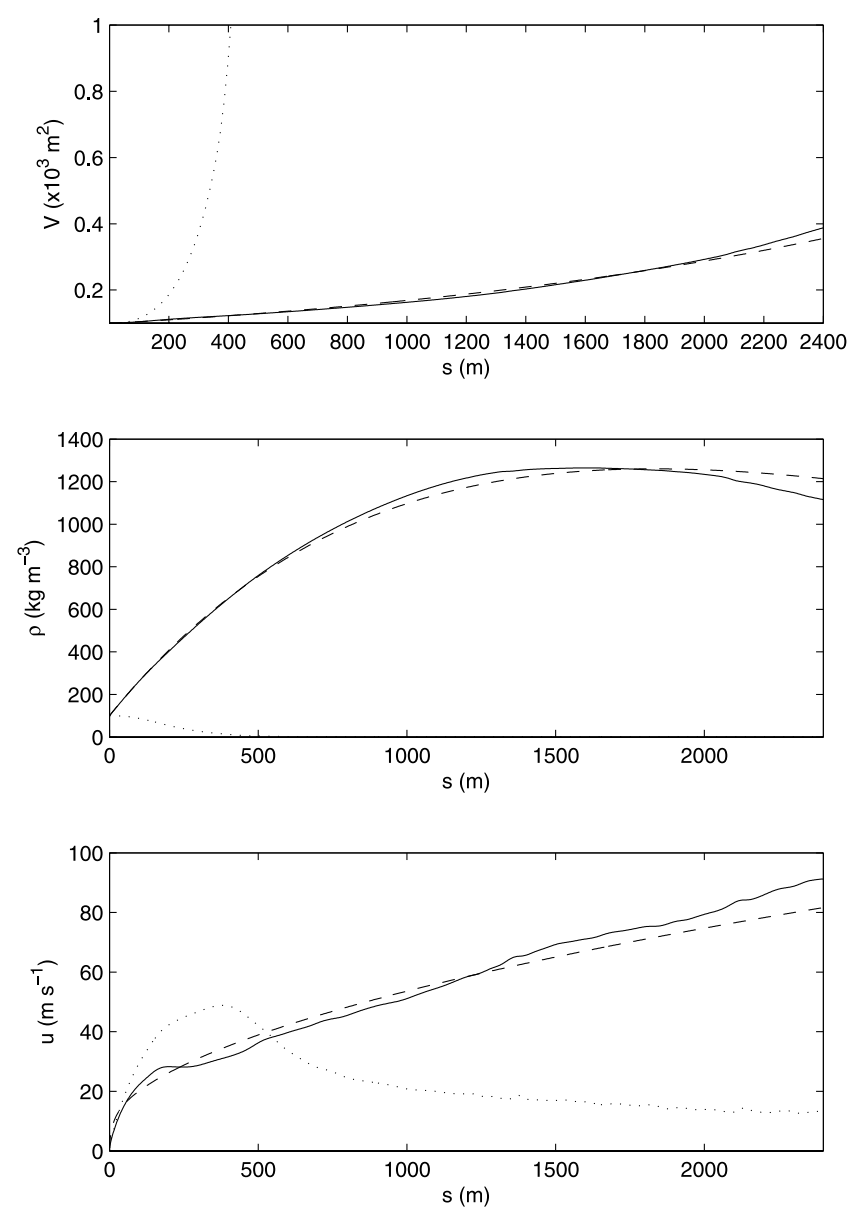

Figure 5. (top) Avalanche volume per unit width, $V$, (middle) powder cloud density, $\rho$, and (bottom) avalanche velocity, $u$, versus front displacement, $s$, for the 25 th February 1999 Vallée de la Sionne avalanche 200, with no snow entrainment (dotted line), with snow entrainment but a flat track (dashed line), and with snow entrainment and spline-smoothed track (solid line).

$\cos \theta, \sin \theta$ and $\tan \theta$ were evaluated directly from the smoothed track as $\frac{\mathrm{d} x}{\mathrm{~d} s}, \frac{\mathrm{d} y}{\mathrm{~d} s}$ and $\frac{\mathrm{d} y}{\mathrm{~d} x}$ respectively. This ensures that $\int_{s 1}^{s 2} \cos \theta \mathrm{ds}=x\left(s_{2}\right)-x\left(s_{1}\right)$, which is not the case if the slope angle $\theta$ is directly interpolated. In this way the distances $s, x$ and $y$ are automatically self-consistent.

[31] Expressions for the growth rates as functions of Richardson number and the aspect ratio $k$ need to be specified. These are taken from Ancey [2004] and listed here.

[32] The overall Richardson number [Turner, 1973] is defined as

$$
\mathrm{Ri}=\frac{\left(\rho-\rho_{a}\right) g h \cos \theta}{\rho_{a} u^{2}},
$$

where the powder cloud height, $h$, is found from the volume per unit width, $V$, and aspect ratio, $k$. For the ellipse in Figure 1

$$
h=2 \sqrt{\frac{k V}{\pi}} .
$$

[33] The volume growth rate $\alpha_{v}$ was found empirically as a function of Richardson number in experiments by Ancey
[2004] (also from the analysis of unpublished data of Beghin). The volume growth rate is fitted by a function

$$
\alpha_{v}=\left\{\begin{aligned}
\mathrm{e}^{-\lambda \mathrm{Ri}^{2}}, & \mathrm{Ri} \leq 1, \\
\mathrm{e}^{-\lambda} / \mathrm{Ri}, & \mathrm{Ri}>1,
\end{aligned}\right.
$$

where $\lambda=1.6$. The Beghin and Ancey experiments cover a small range of Richardson numbers $(0 \lesssim \mathrm{Ri} \lesssim 1.5)$, so care should be taken when applying this empirical function to flows such as developing powder snow avalanches where the Richardson number can be very much higher. For large $\mathrm{Ri}$, the coefficient $\alpha_{v}$ is very small so even if the relative error is large, the error in the entrainment is small.

[34] A function for the aspect ratio, $k$, in terms of the slope angle, $\theta$, in radians was found from the same experiments

$$
k=\left(\gamma_{1}+\gamma_{2} \theta\right)^{\gamma_{3}}
$$

with $\gamma_{1}=0.002155, \gamma_{2}=0.0732$ and $\gamma_{3}=0.3$. As for the analytical solutions, the added mass coefficient $\chi$ is equated with the ellipse aspect ratio, $\chi=k$ (see Appendix A).

[35] Ancey [2004] estimated the entrained snow cover to have a depth of $0.7 \mathrm{~m}$ and density $100 \mathrm{~kg} \mathrm{~m}^{-3}$. However data from photogrammetry measurements shows that this is in fact an underestimation. By subtracting the fracture mass from the mass of deposited snow and dividing by the snow cover density and the area the powder snow avalanche flowed over, the depth can be found as $h_{n}=1.0 \mathrm{~m}$ (to the nearest $0.05 \mathrm{~m}$ ) over the entire track. The density of the snow cover was measured as $\rho_{s}=200 \mathrm{~kg} \mathrm{~m}^{-3}$. Since the $\mathrm{KSB}$ model is not applicable where basal friction is significant, we start the calculation where the density of the fracture slab has halved so that $\rho_{s}=100 \mathrm{~kg} \mathrm{~m}^{-3}$ and the powder cloud has a volume of $100 \mathrm{~m}^{2}$ and velocity $u_{0}=$ $1 \mathrm{~m} \mathrm{~s}^{-1}$. These initial conditions have relatively a minor effect compared with the choice of entrained snow depth and density.

[36] The results of the complete 1999 Vallée de la Sionne avalanche 200 simulation are shown as solid lines in plots of powder cloud volume, density and velocity in Figure 5. Two further numerical calculations were made for the 1999 Vallée de la Sionne avalanche 200: The first, as for the original calculation but with no entrained snow cover $\left(h_{n}=0\right)$; the second, with entrained snow cover as for the original calculation, but with the track profile consisting of only the first and last points, giving a flat slope of the same average slope angle as the Vallée de la Sionne track. These calculations are shown in Figure 5 as dotted and dashed lines respectively. It is noticeable how little difference the track smoothing makes, in particular to the powder cloud velocity and density. The results for the flat slope are very close to those for the spline-smoothed track profile. However, the significant influence of the amount of entrained snow cover is clear in the volume, density and velocity plots.

[37] The calculated velocities match reasonably well with the data acquired from avalanche 200 by Gruber [2004]. Although no density data is available from this avalanche, the KSB model predicts much higher densities than are physically realistic or possible, as the analytical solutions did. These unrealistic densities extend for larger distances 
and are even greater than for the analytical solutions in Figure 2. The main reason for the large densities is that the volume of the entrained snow is not included in the volume equation (1). Another reason why the model predicts such large densities is that the initial conditions used are for a relatively undeveloped powder snow avalanche. By using initial conditions where the powder cloud is developed, the problem might be avoided.

\section{KSB Modified}

[38] One problem with the KSB model as it stands is that the predicted powder cloud densities are unphysically large. Since the model uses a Richardson number-dependent volumetric growth rate, the cloud growth rate is dependent on the cloud density (equation (25)). With poor density predictions, the volume predictions will also be incorrect. At high densities, very little ambient air is entrained, so if the model starts with high densities, it will remain with high densities. The mass of snow entrained from the track is included in the buoyancy equation (5), but its volume is not included in the volume equation (1). This means that the powder cloud densities can become much higher than the density of ice.

[39] By including the volume of the entrained snow mass in the volume equation the densities will be more robust. In this way, realistic densities can be predicted, even when the powder cloud is small. The volume of entrained snow (per unit avalanche width) in a time $\delta t$ can be calculated from equation (5) as $u_{f} h_{n} \delta t$ (this includes the volume of both the ice grains and the air in the pores). So the volume equation (1) becomes

$$
\frac{\mathrm{d} V}{\mathrm{~d} t}=\alpha_{v} u \sqrt{V}+u_{f} h_{n}
$$

[40] An analytical solution for the modified volume (including the volume of entrained snow) can be found, as for the original KSB equations (section 2.1). Nondimensionalized, equation (29) becomes

$$
\frac{\mathrm{d} \tilde{V}}{\mathrm{~d} \tilde{s}}=\alpha_{v}(\sqrt{\tilde{V}}+\delta)
$$

Here, $\delta$ is the ratio between particle and ambient air entrainment $\delta=\eta /\left(1-\tilde{\rho}_{a}\right) \alpha_{v}$, with $\eta /\left(1-\tilde{\rho}_{a}\right)$ the nondimensional effective entrained snow depth, as defined in equation (12). Stronger assumptions are now required to find a simple solution compared with the original analysis in section 2.1. It is necessary to assume $\delta$ is independent of arc coordinate $\tilde{s}$, though $\eta$ and $\alpha_{v}$ are not necessarily independent of $\tilde{s}$. With this assumption, equation (30) can be integrated

$$
\int \frac{\mathrm{d} \tilde{V}^{\prime}}{\sqrt{\tilde{V}^{\prime}}+\delta}=\int \alpha_{v}\left(\tilde{s}^{\prime}\right) \mathrm{d} \tilde{s}^{\prime} .
$$

If $\alpha_{v}$ and $\eta$ are independent of $\tilde{s}$ and for the initial condition $\tilde{V}\left(\tilde{s}_{0 V}\right)=0$ the equation is integrated to give

$$
\sqrt{\tilde{V}}-\delta \ln \left(\frac{\sqrt{\tilde{V}}}{\delta}+1\right)=\frac{\alpha_{v}}{2}\left(\tilde{s}-\tilde{s}_{0 V}\right) .
$$

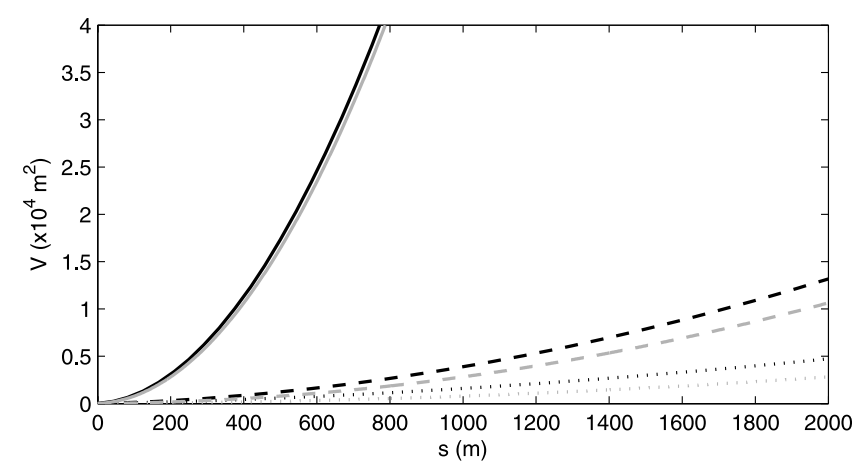

Figure 6. Modified KSB powder cloud volume per unit width, $V$, (including entrained snow volume) versus slope arc position, $s$, for constant snow and air entrainment parameters (equation (33)): $\alpha_{v}=0$. (solid black line), $\alpha_{v}=$ 0.1 (dashed black line), $\alpha_{v}=0.05$ (dotted black line). The gray lines show the same as the black lines but original $\mathrm{KSB}$, without including entrained snow volume.

If the entrainment of ambient fluid is much greater than the entrainment of particles, $\alpha_{v} \gg \eta, \delta$ is small. Expanding the left hand side and neglecting higher-order terms, the original volume solution in equation (18) is recovered. Conversely, if the entrainment of particles is very large compared with the rate of ambient fluid entrainment, $\delta$ is large and the asymptotic expansion of the left hand side, to first order, gives

$$
\tilde{V}=\eta\left(\tilde{s}-\tilde{s}_{0 V}\right)
$$

[41] The volume $\tilde{V}$ from equation (31) can be written explicitly as a function of slope arc coordinate $\tilde{s}$ using a Lambert W (Omega) function; the inverse function of $f(w)=w \mathrm{e}^{w} . W_{-1}(w)$ indicates the negative real branch of the Lambert $\mathrm{W}$ function of $w$ giving

$$
\tilde{V}=\delta^{2}\left[W_{-1}\left\{-\exp \left\{-\left(1+\frac{\left(\tilde{s}-\tilde{s}_{0 V}\right) \alpha_{v}}{2 \delta}\right)\right\}\right\}+1\right]^{2} .
$$

This analytical volume solution is shown in Figure 6 for the initial and ambient conditions listed in Table 2. These conditions give the virtual origins as for the previous analytical solutions given in Table 1. From Figure 6 the inclusion of the entrained snow volume has little effect on the predicted powder cloud volume when $\alpha_{v}$ is sufficiently large but has a significant effect at lower rates of air entrainment.

[42] As in section 2.1 the solution to the modified volume equation (33) and the buoyancy equation (14) can be substituted to find the density variations as a function of slope arc coordinate. The addition of the entrained snow volume makes a significant difference to the powder cloud density and increases the predicted powder cloud volumes. Figure 7 shows the densities predicted by the modified model in comparison with the original model for the initial and ambient conditions in Tables 1 and 2. Although in both models the powder cloud density tends to the density of air after long distances, over distances typical for a powder 


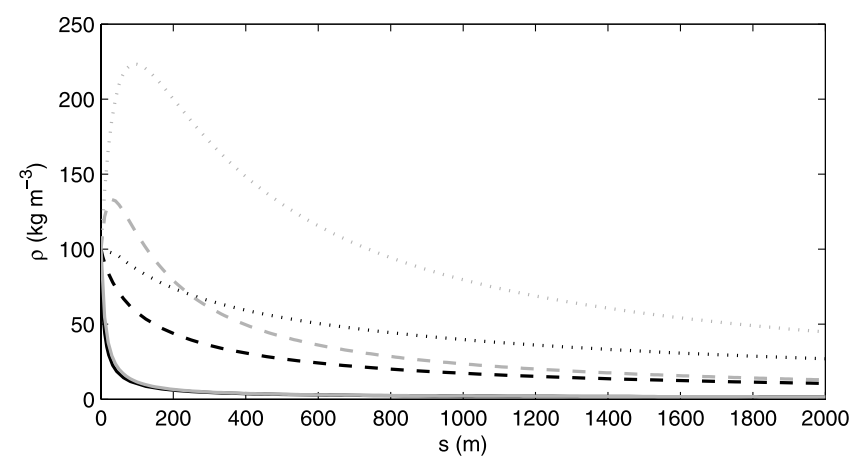

Figure 7. Modified KSB powder cloud density, $\rho$, (including entrained snow volume) versus slope arc position, $s$, for constant snow and air entrainment parameters: $\alpha_{v}=0.5$ (solid black line), $\alpha_{v}=0.1$ (dashed black line), and $\alpha_{v}=0.05$ (dotted black line). The gray lines show the same as the black lines but original KSB, without including entrained snow volume.

snow avalanche the density is significantly reduced by the inclusion of the entrained snow volume.

[43] The velocity solution in equation (17) can be evaluated analytically, as in section 2.1 , since the volumedependent part of the integral can be integrated by parts (the volume solution in equation (33) is integrable twice). This solution has not been shown here since it is unwieldy; however, the velocity function has the same asymptotic properties as the slope arc coordinate $\tilde{s}$ becomes large where the velocity tends to a steady value, equation (24). It can also be shown that with large $\delta$ (i.e., high particle entrainment compared with ambient air entrainment) the predicted velocities are significantly different. In the case of low particle entrainment compared with ambient entrainment, $\delta$ is small and the velocity tends to the original velocity solution, equation (20).

\section{Field Studies}

\subsection{Vallée de la Sionne}

[44] The numerical MATLAB routine introduced in section 2.2 was modified to include the entrained snow volume. Simulations have been rerun with the modified KSB equations from section 3 for the Vallée de la Sionne 1999 avalanche 200, simulated by Ancey [2004] and in section 2.2. The same air entrainment and aspect ratio functions are used as in section 2.2, also the same initial and ambient conditions, listed in Table 2 for avalanche 200.

[45] The simulations in section 2.2 showed that the track smoothing made very little difference to the solutions. Here for simplicity, the first and last points of the slope profile have been used to provide a flat slope.

[46] Plots of the avalanche 200 powder cloud volume, density, and front velocity calculated with the modified KSB model are shown in Figure 8 in solid lines. The dashed lines are the volume, density, and front velocity predicted by the original KSB model, shown for comparison. Points show the front velocity video data from Gruber [2004].

[47] The inclusion of the entrained snow volume makes a significant difference to both the volume and density of the avalanche. Estimating the expected volume of the avalanche is difficult and no conclusion can be drawn from this data as to whether the volume prediction is improved with the modified KSB model. Mean powder cloud densities are expected to be in the range $1 \lesssim \rho \lesssim 150 \mathrm{~kg} \mathrm{~m}^{-3}$ over most of the track, which implies a significant improvement of the modified KSB model compared with the original. The predicted front velocities are also slightly lower with the modified model, giving a very small improvement in the fit with the data.

\subsubsection{Avalanche 509}

[48] Data from the 2003 Vallée de la Sionne avalanche (509) have also been compared with the modified KSB model. Snow entrainment from the track $h_{n}$ was low for avalanche 509 compared with avalanche 200 in section 4.1. By subtracting the fracture mass of snow from the deposited mass, measured from a photogrammetric analysis, and dividing by the entrained snow cover density and the area (i.e. track length and powder snow avalanche width) over which the avalanche ran, the depth of snow entrained across the powder snow avalanche width can be estimated as $0.10 \mathrm{~m}$ (to the nearest $0.05 \mathrm{~m}$ ) [Sovilla, 2004a]. In addition
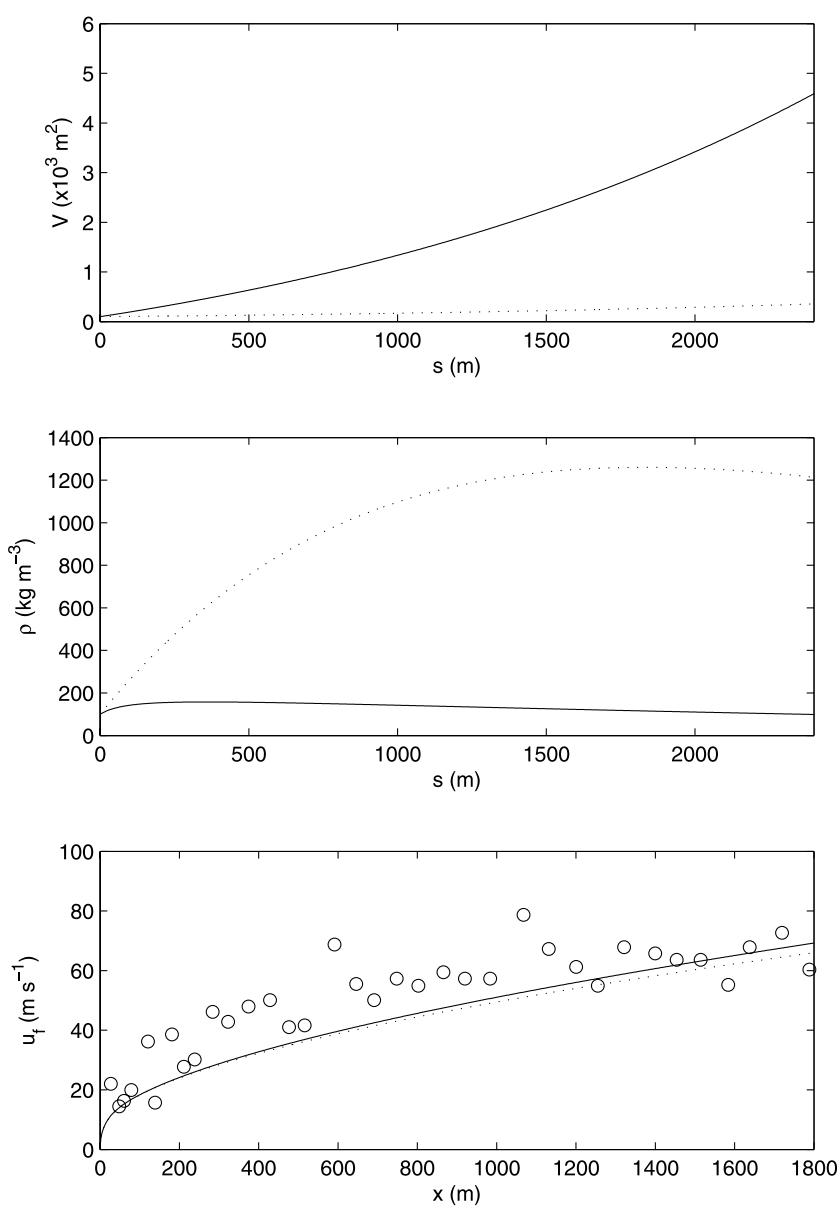

Figure 8. (top) Powder cloud volume per unit width, $V$, versus front displacement, $s$. (middle) Powder cloud density, $\rho$, versus front displacement, $s$. (bottom) Avalanche front velocity, $u_{f}$, versus horizontal displacement, $x$, of the 25 February 1999 Vallée de la Sionne avalanche 200 with a flat slope. KSB modified, solid line; KSB original, dotted line; data points [Gruber, 2004], circles. 

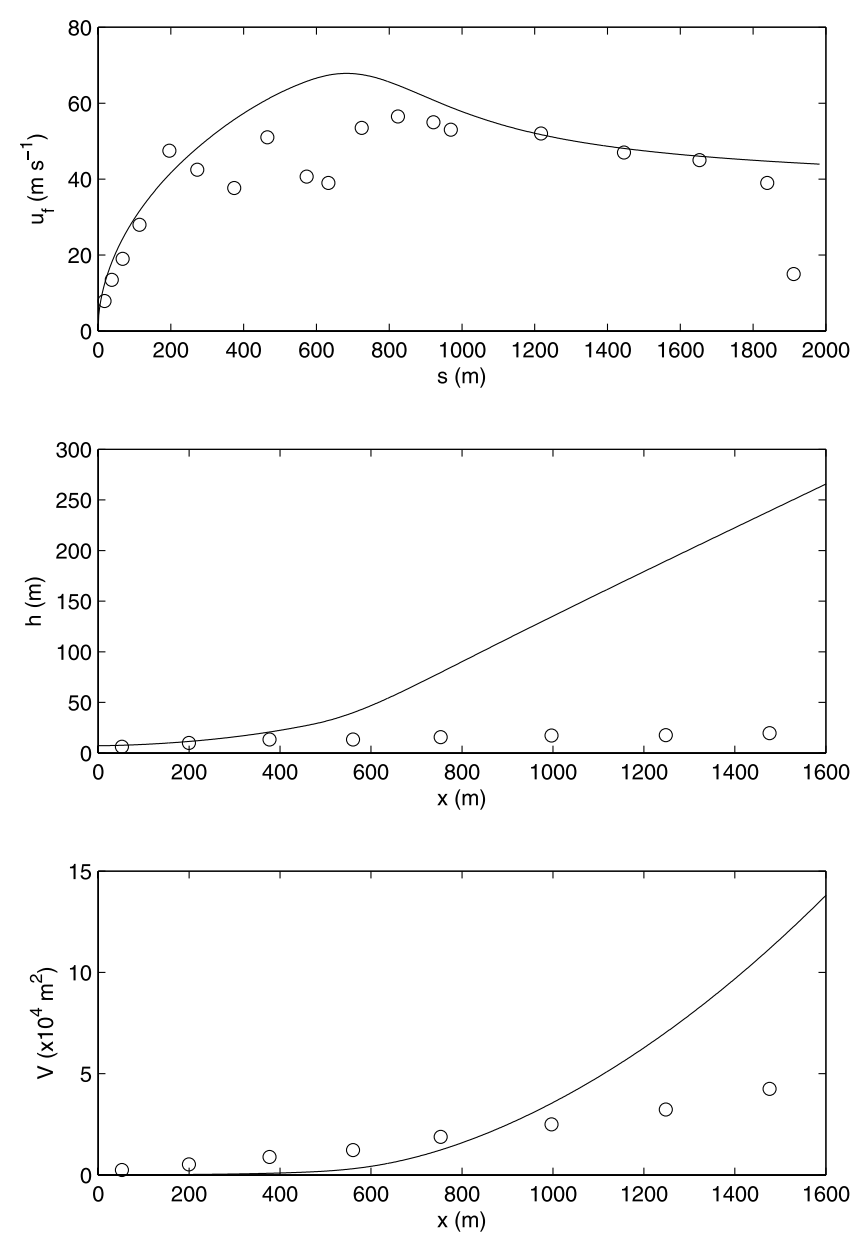

Figure 9. Avalanche 509, powder cloud. (top) Front velocity, $u_{f}$, versus front displacement, $s$. (middle) Height, $h$, versus horizontal displacement, $x$. (bottom) Volume per unit width, $V$, versus horizontal displacement, $x$. Modified KSB model, solid line; videogrammetry data, circles.

the snow cover density was measured during the field test and found to be $195 \mathrm{~kg} \mathrm{~m}^{-3}$. The initial conditions have been kept the same as for avalanche 200 though, as stated previously, the calculation is far more sensitive to the entrainment coefficients along the track than to the initial conditions. Both the initial conditions and entrained snow depth are given in Table 2 for avalanche 509 .

[49] The predicted powder cloud front velocity, height and volume from the modified KSB model are compared in Figure 9 with measurements from the videogrammetry analysis discussed by Turnbull [2006] and Vallet et al. [2004]. With the depth of entrained snow cover $h_{n}=$ $0.10 \mathrm{~m}$, the predicted front velocities fit the data well. The volume data has been divided by the average powder snow avalanche width for comparison with the volume per unit width predicted by the modified KSB model. However, neither the magnitude nor the shape of the predicted powder cloud volumes or heights matches the data. This effect is discussed further in section 4.2.

\subsubsection{Avalanche 628}

[50] The depth of entrained snow cover has been measured, as described for avalanche 509, from the difference in deposited mass and fracture mass measured from photo- grammetry [Sovilla, 2004b]. With the depth of entrained snow cover $h_{n}=0.1 \mathrm{~m}$ and the initial conditions as for the earlier field cases, shown in Table 2, the front velocities of avalanche 628 are well reproduced with the modified KSB model, see Figure 10. For this avalanche there is no flow height data, but particularly for the first part of the track, $(s<1000 \mathrm{~m})$, the cloud heights are lower and slightly more realistic than for avalanche 509. Since both avalanches had the same erosion depths, this height difference is due only to slightly different entrained snow densities and slope angle.

\subsubsection{Avalanche 726}

[51] Only limited data is available for the 2005 Vallée de la Sionne avalanche 726. Importantly, there is no information on the depth of entrained snow. Since this avalanche was of a similar size to nos. 509 and 628 , we will estimate the entrained snow depth, $0.1 \mathrm{~m}$ for both earlier avalanches, to be $0.1 \mathrm{~m}$ also for this avalanche and with a snow density of $200 \mathrm{~kg} \mathrm{~m}^{-3}$. The initial and ambient conditions for the simulation are also kept the same and these are given in Table 2.

[52] The volume per unit width data points in Figure 11 are from the videogrammetry analysis discussed by Turnbull [2006]. To take account of time delay between explosion and avalanche release, and the time taken for the powder snow avalanche to develop from the dense flow, the volume data has been shifted by $-15 \mathrm{~s}$ compared with the simulation. The KSB model predicts the correct magnitude of volume over the course of the avalanche but, as for avalanche 509, the shape of the curve does not provide a convincing fit with data.

\subsection{Discussion}

[53] With the KSB model it is possible to achieve simulations of powder snow avalanches that match well
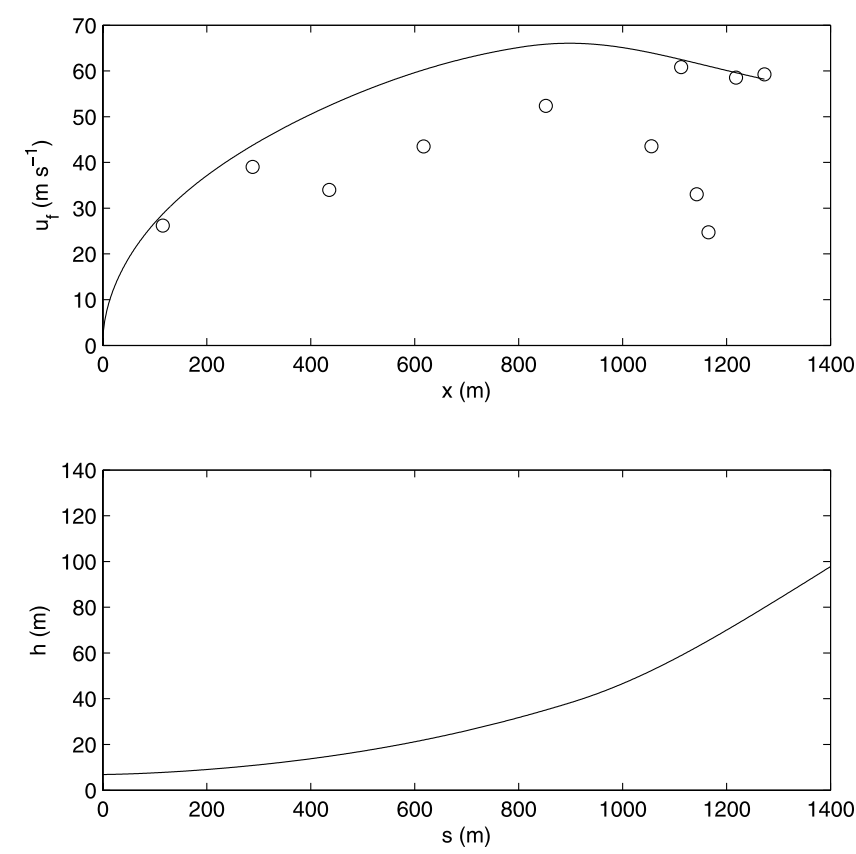

Figure 10. Avalanche 628. (top) Front velocity, $u_{f}$, versus horizontal displacement, $x$. Modified KSB model, solid line; videogrammetry data, circles. (bottom) Powder cloud height, $h$, versus front displacement, $s$, for modified KSB prediction. 


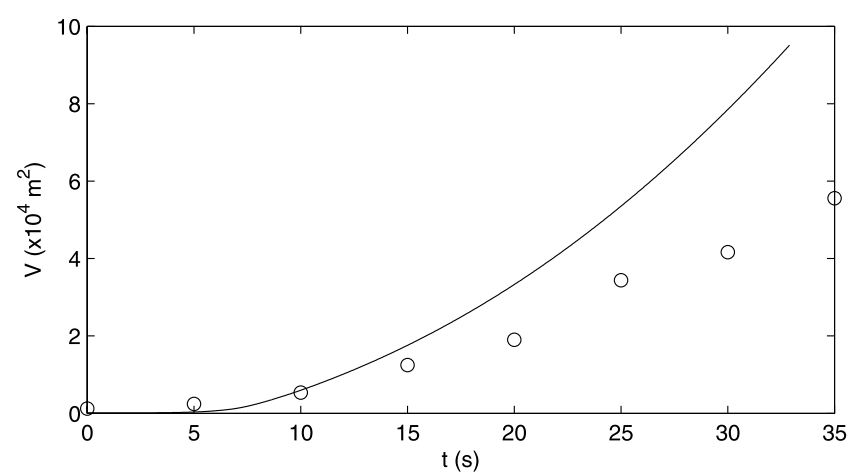

Figure 11. Volume per unit width, $V$ versus time for avalanche 726. Modified KSB model, solid line; videogrammetry data points, circles.

with front velocity data. To provide the necessary information for these simulations, measurements of the depth of snow cover entrained into the avalanche were used. However, such information about the depth and density of entrained snow cover is rare and for simulating previous avalanches the snow entrainment can almost be treated as a free parameter. For practical, predictive applications it would be necessary to develop rules for estimating the entrained snow depth in advance. An additional drawback of integral models such as the KSB model, is that they do not give density and velocity profiles that can be used when calculating possible stagnation pressures.

[54] By including the volume of entrained snow in the KSB volume equation (equation (29)) the powder avalanche densities predicted by the model become far more realistic. Dramatically reducing the densities over a large portion of the track also reduces the Richardson number, allowing increased mixing with the ambient air (equation (27)). However, the model fails to predict correct flow heights and volumes.

[55] The mismatch probably results from treating the avalanche as an ellipse of fixed aspect ratio with constant density and velocity within it. In a real avalanche, the density decreases toward the tail and the tail may reach back as far as the starting zone. The downslope velocity and turbulent intensity will decrease similarly to the density. If the model was extended to allow varying internal densities and velocities parallel to the slope, these defects could possibly be rectified.

[56] In reality some density stratification will also exist perpendicular to the slope, because complete mixing within the cloud will not be achieved. The stratification arises from a combination of particle sedimentation and dilution on the upper surface of the cloud. Introducing a density stratification normal to the slope into the KSB model would change the mixing at the powder cloud-ambient air interface and may be able to better predict the flow heights. Another possibility is to model the turbulent wake separately and to account for fluxes of snow and air between the powder cloud and the turbulent wake.

[57] The KSB model predicts most variables that are dynamically important for a powder snow avalanche such as flow velocity, height and density. One key feature that is not modeled at all is the lateral extent of the avalanche. Currently the model is two-dimensional and though it can be an made into a three-dimensional ellipsoid [Beghin and Olagne, 1991] the next section gives a general derivation of the KSB equations which requires no geometry assumption.

\section{Derivation}

[58] A number of people are critical of simple integral models such as the KSB model described in this paper. Although they are less useful when the topography is complex and has significant variation over scales smaller than the avalanche, they have much wider validity than their critics realize. In this section we show how they can be rigorously derived from the underlying continuum theory. These continuum equations cannot be solved numerically at the appropriate Reynolds number and many closure assumptions are necessary. An advantage of integral models is that fewer assumptions are necessary and those made are straightforward to test directly.

[59] In this section a two-phase mixture approach is adopted where the subscript 1 is used for the snow and 2 is used for the air. $\rho_{i}$ is the density of each species and $\mathbf{u}_{i}$ the velocity. The model can be considered two- or threedimensional. The conservation of mass for each species is then

$$
\frac{\partial \rho_{i}}{\partial t}+\nabla \cdot\left(\rho_{i} \mathbf{u}_{i}\right)=0
$$

and the conservation of momentum is

$$
\frac{\partial \rho_{i} \mathbf{u}_{i}}{\partial t}+\nabla \cdot\left(\rho_{i} \mathbf{u}_{i} \mathbf{u}_{i}\right)+\nabla \sigma_{i}=\mathbf{F}_{i}+\mathbf{g} \rho_{i}
$$

$\sigma_{i}$ is the stress in each species and $\mathbf{F}_{\mathbf{i}}$ are the forces between the species which sum to $\mathbf{0}$. We also require our system to be incompressible. If the volume fraction of snow is $\phi$ then $\rho_{1}=\rho_{1}^{\prime} \phi$ and $\rho_{2}=\rho_{2}^{\prime}(1-\phi)$, where $\rho_{i}^{\prime}$ is the (constant) density of species $i$ at $100 \%$ volume fraction. The incompressibility condition is then

$$
\frac{\rho_{1}}{\rho_{1}^{\prime}}+\frac{\rho_{2}}{\rho_{2}^{\prime}}=\phi+(1-\phi)=1
$$

Combined with the mass conservation equations this shows that the volume weighted velocity is of course divergence free, but the mass weighted velocity field is not.

[60] We integrate these equations over a volume $V$ with surface $S$ that moves with velocity $\mathbf{w}(\mathbf{x})$. The mass of each species $M_{i}=\int \rho_{i} \mathrm{~d} V$ then satisfies

$$
\frac{\mathrm{d} M_{i}}{\mathrm{~d} t}=\int_{S} \rho_{i}\left(\mathbf{w}-\mathbf{u}_{i}\right) \cdot \mathrm{d} \mathbf{n}
$$

For the momentum we use a combined equation and define $M \mathbf{v}=\int\left(\rho_{1} \mathbf{u}_{1}+\rho_{2} \mathbf{u}_{2}\right) \mathrm{d} V$, where $M=M_{1}+M_{2}$. Summing 35 the interspecies forces $\mathbf{F}_{i}$ cancel and we get

$$
\frac{\mathrm{d} M \mathbf{v}}{\mathrm{d} t}=M \mathbf{g}+\int_{S}\left[\rho_{1} \mathbf{u}_{1}\left(\mathbf{w}-\mathbf{u}_{1}\right)+\rho_{2} \mathbf{u}_{2}\left(\mathbf{w}-\mathbf{u}_{2}\right)+\sigma_{1}+\sigma_{2}\right] \cdot \mathrm{d} \mathbf{n} .
$$


[61] Now we specify boundary conditions. On the lower boundary $\left(S_{1}\right)$ only snow is entrained so $\rho_{1}=\rho_{1}^{\prime}$, and $\rho_{2}=0$. The upper boundary $\left(S_{2}\right)$ is taken to be the limit of the snow so that $\rho_{1}=0$ and $\rho_{2}=\rho_{2}^{\prime}$ and $\mathbf{w}=\mathbf{u}_{2}$. Then we get the snow mass equation

$$
\frac{\mathrm{d} M_{1}}{\mathrm{~d} t}=\rho_{1}^{\prime} \int_{S_{1}} \mathbf{w} \cdot \mathrm{d} \mathbf{n}=q_{1},
$$

the air mass equation

$$
\frac{\mathrm{d} M_{2}}{\mathrm{~d} t}=\rho_{2}^{\prime} \int_{S_{2}}\left(\mathbf{u}_{1}-\mathbf{u}_{2}\right) \cdot \mathrm{d} \mathbf{n}=q_{2},
$$

and also a volume equation

$$
\frac{\mathrm{d} V}{\mathrm{~d} t}=\frac{q_{1}}{\rho_{1}^{\prime}}+\frac{q_{2}}{\rho_{2}^{\prime}},
$$

which agrees with the integrated incompressibility constraint

$$
V=\frac{M_{1}}{\rho_{1}^{\prime}}+\frac{M_{2}}{\rho_{2}^{\prime}} .
$$

The fluxes in the momentum equation on the lower surface vanish since $\mathbf{u}_{i}=\mathbf{0}$ in the snowpack. On the upper surface $\rho_{1}$ and $\sigma_{1}$ vanish. Up until this point our system is exact, but now we must make assumptions to proceed. First of all we ignore all surface tractions, that is we assume that the surfaces stresses can be represented by a pressure $p=$ $\sigma_{1}+\sigma_{2}$. We further assume that this has three components. A background constant $p_{0}$, a hydrostatic component $\mathbf{x} \cdot \mathbf{g} \rho_{2}$ and an added mass component that will contribute $-\frac{\mathrm{d}\left(\chi V \rho_{2}^{\prime} \mathbf{v}\right)}{\mathrm{d} t}$ after integration. This added mass contribution is also assumed to account for the momentum flux of air on the upper surface $\mathbf{u}_{2} \rho_{2}\left(\mathbf{u}_{1}-\mathbf{u}_{2}\right) \cdot \mathrm{d} \mathbf{n}$. Next we restrict ourselves to just considering the downslope component of the momentum, $M v$, and assume that the slope is flat over the size of the avalanche. Then we get

$$
\frac{\mathrm{d} v\left(M+\chi V \rho_{2}^{\prime}\right)}{\mathrm{d} t}=\left(M-V \rho_{2}^{\prime}\right) g \sin \theta,
$$

which can be written

$$
\frac{\mathrm{d} v\left[B+(1+\chi) V \rho_{2}^{\prime}\right]}{\mathrm{d} t}=B g \sin \theta,
$$

where $B=M-V \rho_{2}{ }^{\prime}$ is the buoyancy. We can combine the mass equation with the volume equation to regain our original KSB formulation

$$
\frac{\mathrm{d} B}{\mathrm{~d} t}=q_{1}\left(1-\frac{\rho_{2}}{\rho_{2}^{\prime}}\right)=q_{1}^{\prime} .
$$

[62] What this derivation shows is that the KSB model is much more general than in its original formulation. This approach also shows how it is straightforward to account for gentle slope curvature and surface tractions. The difficult assumptions relate to the flux of air and its momentum on the upper boundary. Assuming that the entrained air has zero momentum is a large assumption, but this error may be partially canceled out by the assumption that the dynamic pressure distribution integrates to zero over the surface, which is certainly not true since flow separation will occur. Thus the pressure behind the avalanche will be close to the ambient pressure and the difference between this and the high pressures on the front surface will give rise to form drag. Another approach avoids these difficulties by integrating to infinity as is common in plume theories. In this approach the volume is defined by an integral $V \mathbf{v}=\int \mathbf{v d} V$, thus it explicitly includes all the momentum of the air so there is no added mass effect and no pressure forces on the upper surface to consider. The drawback of this approach is that it is then hard to say what volume $V$ corresponds to and how this should be compared with measurements and so a time evolution equation for it must be posited rather than derived. Thus the problems are all shifted on to choosing the appropriate volume flux function. By explicitly including separate velocity fields for the snow and air, sedimentation on the upper and lower surface can also be included, which is important in the initiation phase and the sedimentation phase when the velocity decreases. The geometry assumption in the KSB model affects the air entrainment function, but also the relation between the front of the avalanche and the evolution of the center of mass. This model could be extended to include more details of the internal structure by having equations for length, height and width instead of volume, but this is beyond the scope of this paper, and is best done in conjunction with laboratory experiments.

\section{Conclusions}

[63] In this work the Kulikovskiy-Sveshnikova-Beghin (KSB) equations describing the motion of a particle cloud on an incline have been introduced and extended. Analytical solutions have been found for the case of constant entrainment parameters and a constant slope angle. The equations have also been solved for a large powder snow avalanche measured at the Vallée de la Sionne test site. For both the analytical solutions and the field case, the powder cloud densities predicted by the KSB model were unrealistically large. Since the volume growth rate is a function of Richardson number, which itself is a function of the powder cloud density, these unrealistic densities affect the ambient mixing and thus the volume and front velocity predictions of the model.

[64] Analytical solutions for modified KSB equations have been found which include the volume of the entrained snow in the volume equation. These modified solutions give improved and physically realistic predictions of powder cloud density. Improved powder cloud density and velocity predictions are found for several field cases, however the size and shape of the avalanche are still not well modeled.

[65] The KSB model provides a simple and reliable method of predicting avalanche velocities. Solutions are sensitive to the depth of entrained snow cover, but the smoothness of the track has little effect. Particle deposition is not modeled at all, making the model invalid in its decelerating phase. However, with the inclusion of the entrained snow volume, the modified equations can be 
applied to the early stages of a powder snow avalanche so long as the dominating drag force arises from the acceleration of entrained matter.

[66] Further work lies in extending the applicability of the KSB model. This can be done by attempting to model particle entrainment and deposition more rigorously. Methods could also be explored for making the model more representational, in terms of shape, of what is observed in an avalanche. For example, the turbulent wake and avalanche head could be modeled separately. By deriving the KSB equations from the underlying continuum theory, it is shown how increasing degrees of complexity may be simply incorporated into the KSB model; for example varying geometries or the effects of stratification.

\section{Appendix A: Flow Around an Ellipse}

[67] The steady, inviscid, incompressible flow with speed 1 around an ellipse of radius 1 in the flow direction and $\kappa$ orthogonally is described by the complex potential

$$
w=-\frac{\kappa(1+\kappa)}{z+\sqrt{z^{2}+\kappa^{2}-1}},
$$

where $z=x+i y, \phi=\operatorname{Re} w$ is the velocity potential and $\psi=$ $\operatorname{Im} \psi$ is the stream function. The surface of the ellipse is described by

$$
x=\cos \theta, \quad y=\kappa \sin \theta
$$

The added mass can be defined as the mass of a body, $M_{a}$, that would have the same kinetic energy as the fluid if it moved with the velocity of the body. Thus

$$
M_{a}=\int_{A}(\nabla \phi)^{2} \mathrm{~d} A^{\prime}
$$

where the integral is over all space, $A$, outside the ellipse. Since the integrand is nonsingular, strongly vanishes at infinity and satisfies Laplace's equation we can use Gauss' theorem to get an integral over the circumference of the ellipse, so

$$
\begin{aligned}
M_{a} & =\int-\phi(\nabla \phi) \cdot \hat{\mathbf{n}} \mathrm{d} s \\
& =\int-\phi(\hat{\mathbf{x}} \cdot \hat{\mathbf{n}}) \mathrm{d} s
\end{aligned}
$$

Now on the ellipse $\phi=\operatorname{Re} w=\operatorname{Re}-\kappa e^{i \theta}=-\kappa \cos \theta$, so

$$
\begin{aligned}
M_{a} & =\int_{0}^{2 \pi} \kappa \cos \theta \frac{\kappa \cos \theta}{\sqrt{\kappa^{2} \cos ^{2} \theta+\sin ^{2} \theta}} \sqrt{\kappa^{2} \cos ^{2} \theta+\sin ^{2} \theta} \mathrm{d} \theta \\
& =\int_{0}^{2 \pi} \kappa^{2} \cos ^{2} \theta \mathrm{d} \theta \\
& =\kappa^{2} \pi
\end{aligned}
$$

Since the area of the ellipse $V=\pi \kappa$ the added mass coefficient is $M_{a} / V=\kappa$.
[68] Acknowledgments. We would like to thank M. M. Scase for many useful discussions and also both reviewers, in particular Dieter Issler, for their constructive and detailed comments. B.T. was funded by the WSL, Swiss Federal Institute for Snow and Avalanche Research (SLF). J.N.M. was funded by the EU SATSIE project (contract EVGI-CT2002-00059), EPSRC (GR/TO2416101) and the Royal Society.

\section{References}

Ancey, C. (2004), Powder-snow avalanches: Approximation as nonBoussinesq clouds with a Richardson number-dependent entrainment function, J. Geophys. Res., 109, F01005, doi:10.1029/2003JF000052.

Batchelor, G. (1967), An Introduction to Fluid Dynamics, Cambridge Univ. Press, New York.

Beghin, P. (1979), Etude des bouffées bidimensionnelles de densité en écoulement sur pente avec application aux avalanches de neige poudreuse, Ph.D. thesis, Inst. Natl. Polytech. de Grenoble, Grenoble, France.

Beghin, P., and X. Olagne (1991), Experimental and theoretical study of the dynamics of powder snow avalanches, Cold Reg. Sci. Technol., 19, 317326.

Boussinesq, J. (1903), Théorie Analytique de la Chaleur, vol. 2, GauthierVillars, Paris.

Bozhinskiy, N., and K. Losev (1998), Fundamentals of Avalanche Science, translated from Russian by C. E. Bartelt, Eidg. Inst. für Schnee- und Lawinenforschung, Davos, Switzerland.

Dufour, F., U. Gruber, P. Bartelt, and W. Ammann (2000), Overview of the 1999 measurements at the SLF test site, Vallée de la Sionne, paper presented at ISSW 2000: A Merging of Theory and Practice, Int. Snow Sci. Workshop, Big Sky, Mont.

Ellison, T., and J. Turner (1959), Turbulent entrainment in stratified flows, J. Fluid Mech., 6, 423-448.

Fukushima, Y., and G. Parker (1990), Numerical simulation of powder snow avalanches, J. Glaciol., 36, 229-237.

Gauer, P. (1995), A model of powder snow avalanches, in Les Apports de la Recherche Scientifique á la Sécurité Neige, Glace et Avalanche, Actes de Colloque, pp. 55-61, Int. Comm. for Alp. Rescue, Chamonix, France.

Gruber, U., et al (2004), Avalanche dynamics experimental site Vallée de la Sionne: Final report winter 1998/1999, Tech. Rep. 732, Swiss Fed. Inst. for Snow and Avalanche Res., Davos.

Hogg, A., and A. Woods (2001), The transition from inertia to bottom drag dominated motion of a turbulent gravity current, J. Fluid Mech., 449, $201-224$.

Issler, D. (1998), Modelling of snow entrainment and deposition in powdersnow avalanches, Ann. Glaciol., 26, 253-258.

Kulikovskiy, A., and E. Sveshnikova (1977), Model dlja rascheta dvizhija pilevoi snezhnoi lavini (A model for computing powdered snow avalanche motion), Mat. Glatsiologicheskih Isseledovanii, 31, 74-80.

Naaim, M., and I. Gurer (1998), Two-phase numerical model of powder avalanche: Theory and application, J. Nat. Hazards, 117, 129-145.

Riley, K., M. Hobson, and S. Bence (1997), Mathematical Methods for Physics and Engineering, Cambridge Univ. Press, New York.

Sampl, P. (1993), Current status of the AVL avalanche simulation modelNumerical simulation of dry snow avalanches, in International Workshop on Gravitational Mass Movements, edited by L. Buisson and G. Brugnot, pp. 269-278, Cemagref Ed., Antony, France.

Scheiwiller, T., K. Hutter, and F. Hermann (1987), Dynamics of powder snow avalanches, Ann. Geophys., Ser. B, 5, 569-588.

Sovilla, B., et al (2004a), Avalanche dynamics experimental site Vallée de la Sionne: Final report winter 2002/2003, Tech. Rep. 750, Swiss Fed. Inst. for Snow and Avalanche Res., Davos.

Sovilla, B., et al (2004b), Avalanche dynamics experimental site Vallée de la Sionne: Final report winter 2003/2004, Tech. Rep. 753, Swiss Fed. Inst. for Snow and Avalanche Res., Davos.

Turnbull, B. (2006), Dynamics of powder snow avalanches, Ph.D. thesis, Univ. of Dundee, Dundee, U. K.

Turner, J. (1973), Buoyancy Effects in Fluids, Cambridge Univ. Press, New York.

Vallet, J., B. Turnbull, S. Joly, and F. Dufour (2004), Observations on powder snow avalanches using videogrammetry, Cold Reg. Sci. Technol., $39,153-159$.

C. Ancey, Ecole Polytechnique Fédérale de Lausanne, CH-1015 Lausanne, Switzerland.

J. N. McElwaine, Department of Applied Mathematics and Theoretical Physics, University of Cambridge, Cambridge CB2 1TN, UK.

B. Turnbull, DAMTP, University of Cambridge, CMS, Wilberforce Road, Cambridge CB3 0WA, UK. (b.turnbull@damtp.cam.ac.uk) 